\title{
Details of Magnetic Properties in $\mathrm{Pb}\left(\mathrm{Fe}_{1 / 2} \mathrm{Nb}_{1 / 2}\right) \mathrm{O}_{3}$
}

\author{
M. MARYŠKO ${ }^{a} *$, V.V. LAGUTA ${ }^{a}$, I.P. RAEVSKI ${ }^{b}$ \\ ${ }^{a}$ Institute of Physics of ASCR, Na Slovance 2,18200 Prague 8, Czech Republic \\ ${ }^{b}$ Institute of Physics, Southern Federal University, Rostov on Don 344090, Russia
}

\begin{abstract}
For $\mathrm{Pb}\left(\mathrm{Fe}_{0.5} \mathrm{Nb}_{0.5}\right) \mathrm{O}_{3}$ the $\chi(T)$ and $m(H)$ measurements up to $70 \mathrm{kOe}$ were performed with the aim to estimate the antiferromagnetic (AFM) and superantiferromagnetic (SAF) contribution to $\chi(T)$. Below $T_{\mathrm{N}}$ the increase of $\chi(T)$ is attributed to a small part of $\mathrm{Fe}^{3+}$ ions in the SAF clusters. For high fields the suppression of the AFM and enhancement of the SAF susceptibility was observed. An inflection point of the $m(H)$ curves below the Néel point suggests the presence of a spin reorientation process connected with the AFM phase.
\end{abstract}

DOI: 10.12693 /APhysPolA.126.376

PACS: 75.30.Cr, 75.85.+t, 75.50.Ee

\section{Introduction}

Lead iron niobate $\mathrm{Pb}\left(\mathrm{Fe}_{0.5} \mathrm{Nb}_{0.5}\right) \mathrm{O}_{3}(\mathrm{PFN})$ and the $\mathrm{PFN}$ based solid solutions have been studied in recent years with regard to their extreme multiferroic properties [1-7]. The basic magnetic properties of PFN were reported many years ago [8] but because of their complexity they were not up to now consistently interpreted. Above the Néel temperature $T_{\mathrm{N}} \approx 145 \mathrm{~K} \mathrm{PFN}$ is a paramagnet $(\mathrm{PM})$ and below $T_{\mathrm{N}}$ this compound seems to represent a mixture of the long-range antiferromagnetic (AFM) and superantiferromagnetic (SAF) $\mathrm{Fe}^{3+}$ clusters [4]. At low temperatures a cluster glass $(\mathrm{CG})$ state is observed with a freezing temperature near $10 \mathrm{~K}[1,2,4,7]$. In the present contribution, following the work on $\mathrm{Ba}$ and $\mathrm{Ti}$ doped PFN [7], we are concerned with the more detailed magnetometric study of PFN with the aim to clarify the increase of the susceptibility below $T_{\mathrm{N}}$ when going towards lower temperatures.

\section{Experimental}

The experiments were made on non-oriented single crystals of $\mathrm{Pb}\left(\mathrm{Fe}_{0.5} \mathrm{Nb}_{0.5}\right) \mathrm{O}_{3}$ grown by the spontaneous crystallization procedure from the $\mathrm{PbO}-\mathrm{B}_{2} \mathrm{O}_{3}$ flux (for details we refer to the work [7]). The magnetic measurements were carried out using the SQUID magnetometers MPMS-5S, MPMS-XL (Quantum Design). The zerofield (ZFC) and field-cooled (FC) susceptibilities $\chi_{\mathrm{ZFC}}$, $\chi_{\mathrm{FC}}$ were measured under different magnetic fields up to $70 \mathrm{kOe}$. The magnetization curves $m(H)$ with increasing field (virgin curves) and decreasing field were recorded in the wide range of temperatures ( $m$ is expressed in Bohr magnetons p.f.u.). The irreversibility has been found only below $10 \mathrm{~K}$, which is in accord with the temperature dependence of the remanence [7].

Letting aside $\chi_{\mathrm{ZFC}}$ and problems of the $\mathrm{CG}$ state we focus our attention on the $\chi_{\mathrm{FC}}$ measured under different applied fields. The transition from a PM to long-range AFM state at the Néel temperature $T_{\mathrm{N}}$ can be well seen on the derivative of the $d \chi_{\mathrm{FC}} / d T$. This dependence for

*corresponding author; e-mail: marysko@fzu.cz
$H=500$ Oe is shown in Fig. 1a. The amplitude $A$ of $\mathrm{d} \chi_{\mathrm{FC}} / \mathrm{d} T$ depends on $H$ (inset of Fig. 1a). For $H=$ $70 \mathrm{kOe}$ the transition is still visible but the amplitude $A$ decreases to about 0.01 of the value at the low fields. In Fig. 1b we show $\mathrm{d}\left(1 / \chi_{\mathrm{FC}}\right) / \mathrm{d} T$ for $H=500$ Oe and $H=70 \mathrm{kOe}$. The results of the study of the $m(H)$ curves are summarized in Fig. 2, in the form of the derivatives $\mathrm{d} m / \mathrm{d} H$. At $T=2 \mathrm{~K}$ and between 60 and $135 \mathrm{~K}$ we find a maximum of $\mathrm{d} m / \mathrm{d} H$ (inflection point of $m(H)$ ) at a critical field $H_{\mathrm{inf}}$ depending on temperature (inset of Fig. 2b).

\section{Discussion}

In the low temperature region $(2-40 \mathrm{~K})$ (Fig. $2 \mathrm{~b}$ ) we find an inflection point of the $m(H)$ at $T=2 \mathrm{~K}$, which is a typical feature of the CG state (an $\mathrm{S}$ type of the $m(H))$. Near the freezing temperature, at $T=10 \mathrm{~K}$ this behaviour is absent.

Below $T_{\mathrm{N}}$ and reliably outside the region of the cluster growth $\left(T<T_{g s} \approx 50 \mathrm{~K}[7]\right)$, between 60 and $120 \mathrm{~K}$ the $\chi_{\mathrm{FC}}(T)$ should contain both AFM and SAF contributions. The first $\chi_{\mathrm{AFM}}$ has its origin in the long-range AFM (proved by the neutron diffraction in the work [3]) and the second SAF contribution $\chi_{\mathrm{SAF}}(T)$ corresponds to uncompensated spins. In our case, we may assume that $\chi_{\mathrm{SAF}}(T)$ follows the Curie-Weiss $(\mathrm{CW})$ law (generally is given by the Brillouin function). An attempt to crudely estimate the value of these two contributions for $H=500$ Oe will be made using a simplified assumption that between 60 and $120 \mathrm{~K} \chi_{\mathrm{AFM}}$ does not depend on temperature. Starting then from the relation $\chi_{\mathrm{FC}}=$ $\chi_{\mathrm{AFM}}+C /(T+\theta)$ we get $C=0.328 \mathrm{emu} \mathrm{K} /(\mathrm{mol} \mathrm{Oe})$, $\theta=14.8 \mathrm{~K}$ and $\chi_{\mathrm{AFM}}=0.0019 \mathrm{emu} /(\mathrm{mol} \mathrm{Oe})$. This AFM susceptibility representing about one half of the expected $\chi_{\mathrm{AFM}}$ at the Néel point seems to be reasonable. (Taking $\theta=520 \mathrm{~K}[8]$ we obtain $\chi_{\mathrm{AFM}}(145 \mathrm{~K})=$ $0.0032 \mathrm{emu} /(\mathrm{mol} \mathrm{Oe}))$. The values of $d(1 / \chi) / d T$ corresponding to this approximation are denoted in Fig. 1b by open circles. The interesting fact concerns the influence of the magnetic field. We see that for $H=70 \mathrm{kOe}$, between 100 and $140 \mathrm{~K}$ the value $\mathrm{d}\left(1 / \chi_{\mathrm{FC}}\right) / \mathrm{d} T$ remains approximately constant (Fig. 1b), which corresponds to the situation that $\chi_{\mathrm{FC}}(T)$ is given only by a $\mathrm{CW}$ term. In this case we obtain $C=0.9 \mathrm{emu} \mathrm{K} /(\mathrm{mol} \mathrm{Oe})$ and $\theta=90.4 \mathrm{~K}$. 


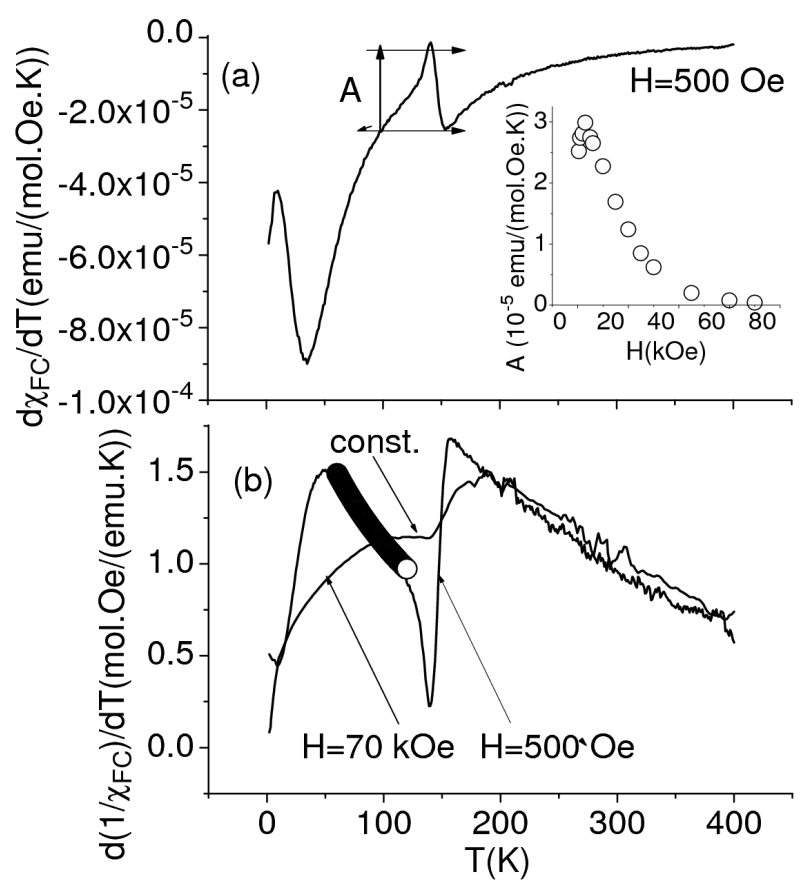

Fig. 1. (a) Evaluated values of $\mathrm{d} \chi_{\mathrm{FC}} / \mathrm{d} T$ for $H=$ $500 \mathrm{Oe}$, in the inset: amplitude $\mathrm{A}$ of $\mathrm{d} \chi_{\mathrm{FC}} / \mathrm{d} T$ at $T=T_{\mathrm{N}}$ as a function of the applied field, (b) evaluated values of $\mathrm{d}\left(1 / \chi_{\mathrm{FC}}\right) / \mathrm{d} T$ for the applied fields $500 \mathrm{Oe}$ and $70 \mathrm{kOe}$; open circles denote the approximation with $\chi_{\mathrm{AFM}}$ independent on temperature.

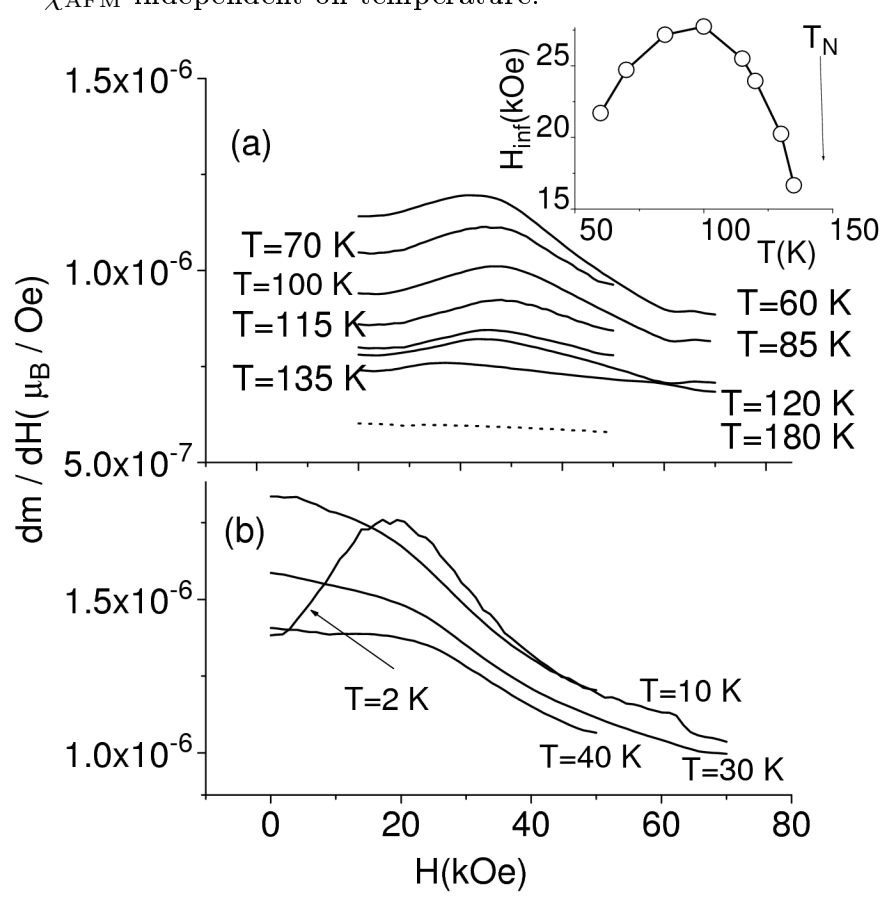

Fig. 2. Evaluated values of $\mathrm{d} m / \mathrm{d} H$, (a) the temperature region $60-180 \mathrm{~K}$, in the inset: the field $H_{\text {inf }}$ corresponding to the inflection point of $m(H)$ as a function of temperature, (b) temperature region 2-40 K.

This suggests that the magnetic field suppresses the AFM and enhances the SAF contribution. The same conclusion follows from the observed essential decrease of the amplitude $A$ with increasing field. The reason for this behaviour remains unclear.

The portion $p$ of the $\mathrm{Fe}^{3+}$ ions contributing to $\chi_{\mathrm{SAF}}$ can be determined as $8.75 C /\left(2.1875 S_{1}\left(S_{1}+1\right)\right)$ with $S_{1}$ denoting a spin of the uncompensated magnetic moment. (For $0.5 \mathrm{Fe}^{3+}$ the Curie constant $C=$ $2.1875 \mathrm{emu} \mathrm{K} /(\mathrm{mol} \mathrm{Oe}))$.

The behaviour of $\mathrm{d} m / \mathrm{d} H$ (Fig. 2) suggests that $\chi_{\mathrm{SAF}}$ is caused by small clusters with $S_{1}$ of the order of tens. In this case the increase of the $\chi_{\mathrm{FC}}$ below $T_{\mathrm{N}}$ can be ascribed to only a small portion (several percents) of the $\mathrm{Fe}^{3+}$ ions. The field dependence of $\mathrm{d} m / \mathrm{d} H$ has its origin in two contributions. The major contribution is due to the Brillouin behaviour of SAF clusters and second, with a maximum in $\mathrm{d} m / \mathrm{d} H$, can be ascribed to a spin reorientation in the AFM phase. With regard to the high value of the molecular field corresponding to $T_{\mathrm{N}}\left(10^{6} \mathrm{Oe}\right)$, this process seems not to be a metamagnetic transition in the long-range AFM phase, but rather is connected with a spin-flop transition.

\section{Conclusions}

At low fields ( $H=500$ Oe) the susceptibility below $T_{\mathrm{N}}(60-120 \mathrm{~K})$, increasing with decreasing temperature, could be attributed to several percents of $\mathrm{Fe}^{3+}$ ions in the SAF clusters. For $H=70 \mathrm{kOe}$, below $T_{\mathrm{N}}$ the susceptibility seems to be formed only by a SAF term the AFM contribution being suppressed. In the temperature region $60-135 \mathrm{~K}$ the occurrence of an inflection point on the $m(H)$ curve indicates the presence of a spin-reorientation process connected with the AFM phase.

\section{Acknowledgments}

This work was supported by the project No. 13-11473S of the Grant Agency of the Czech Republic.

\section{References}

[1] A. Falqui, N. Lampis, A. Geddo-Lehmann, G. Pinna, J. Phys. Chem. B 109, 22967 (2005).

[2] A. Kumar, R.S. Katiyar, C. Rinaldi, S.G. Lushnikov, T.A. Shaplygina, Appl. Phys. Lett. 93, 232902 (2008).

[3] G.M. Rotaru, B. Roessli, A. Amato, S.N. Gvasaliya, C. Mudry, S.G. Lushnikov, T.A. Shaplygina, Phys. Rev. B 79, 184430 (2009).

[4] W. Kleeman, V.V. Shvartsman, P. Borisov, A. Kania, Phys. Rev. Lett. 105, 257202 (2010).

[5] R. Havlíček, J. Poltierová Vejpravová , D. Bochenek, J. Phys.: Conf. Ser. 200, 012058 (2010).

[6] I.P. Raevski, S.P. Kubrin, S.I. Raevskaja, D.A. Sarychev, S.A. Prosandeev, M.A. Malitskaya, Phys. Rev. B 85, 224412 (2012).

[7] V.V. Laguta, M.D. Glinchuk, M. Maryško, R.O. Kuzian, S.A. Prosamdeev, S.I. Raevskaya, V.G. Smotrakov, V.V. Eremkin, I.P. Raevski, Phys. Rev. B 87, 064403 (2013).

[8] V.A. Bokov, I.E. Mylnikova, G.A. Smolenskii, Sov. Phys. JETP 15, 447 (1962). 